Short Report

\title{
Single Center Experience in Patients with A Diagnosis of Nodular Lymphocyte Predominant Hodgkin Lymphoma
}

\author{
Ömer CANDAR ${ }^{1}$, Fahir ÖZKALEMKAŞ ${ }^{1}$, Vildan ÖZKOCAMAN ${ }^{1}$, Tuba ERSAL ${ }^{1}$, İbrahim Ethem \\ PINAR ${ }^{1}$, Cumali YALÇIN ${ }^{1}$, Bedrettin ORHAN ${ }^{1}$, Rıdvan ALI ${ }^{1}$ \\ ${ }^{1}$ Bursa Uludağ University Medical Faculty, Department of Internal Medicine, Division of Hematology, Bursa, Turkey
}

Turk J Int Med 2021;3(Supplement 1):S40-S41

DOI: $\underline{10.46310 / \text { tjim.877049 }}$

Keywords: Nodular lymphocyte predominant Hodgkin lymphoma, Hodgkin lymphoma, prognosis

Nodular Lymphocyte Predominant Hodgkin Lymphoma (NLPHL) is a rare hematological malignancy with an excellent prognosis. It is about $5 \%$ of Hodgkin lymphoma cases. The incidence of the disease is $8-9$ per 10 million. In this study, we planned to evaluate the clinical features and prognosis of patients diagnosed with NLPHL in the last ten years followed in the hematology department.

In our study, the data of 10 patients who were diagnosed with NLPHL between January 2010 and December 2020 in Bursa Uludag University Faculty of Medicine, Department of Hematology, were evaluated retrospectively. $2.2 \%$ of 450 Hodgkin lymphoma-diagnosed patients were diagnosed with NLPHL, in 10 years. $80 \%$ of patients were male. Median age was found 36 years (28-60). At the time of diagnosis, all patients had ECOG score of 0 and $80 \%$ of the patients were at early stage. According to International Prognostic Score(IPS), patients were low risk. All patients who applied to the hospital, complained about palpable lymphadenopathy at first. None of the patients had B symptoms. Hemograms and biochemical parameters were in reference intervals at the time of diagnosis. Immunohistochemical staining of the biopsies showed that all specimens were positive for CD20, but just one of them positive for CD30. Eight patients were administered ABVD (doxorubicin, bleomycin, vinblastine, dacarbazine) chemotherapy as first-line therapy. On the other hand, two patients received coadministration of radiotherapy (RT) with ABVD. Two patients who were refractory first-line therapy, took DHAP (dexamethasone, cytarabine, cisplatin) as salvage chemotherapy regimen. Patients, completed remission after treatment, had autologous stem cell transplant (ASCT) after completion of BEAM (carmustine, etoposide, cytarabine, melphalan) conditioning regimen. One of our transplant patients recurred 5 months after the transplant. Gemcitabine chemotherapy was given to the patient who developed recurrence. He died due to sepsis in the third cycle of the treatment and in the 10 th month of the transplant. Other patient is still alive and followed up in remission. Kaplan Meier

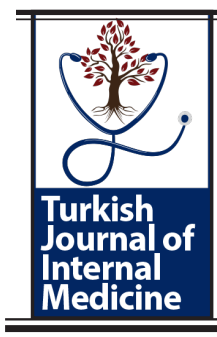

Received:February 18, 2021;Accepted:March 5, 2021;Published Online:March 6, 2021

Address for Correspondence:

Ömer CANDAR

Bursa Uludağ University Faculty of Medicine, Department of Internal Medicine, Division of

Hematology, Bursa, Turkey

E-mail:oeml6365@gmail.com 
survival analysis computed four-year survival rate $80 \%$. Overall survival could not be estimated. Median survival time was 42.2 months.

NLPHL is a less common disease that differs from classical Hodgkin lymphoma in histology, course, and treatment. It is associated with a favorable prognosis. The most important problems with the management of the disease are relapses, transformation to non-Hodgkin lymphoma, and treatment-related toxicities. There are no randomized controlled trials with its treatment due to its rarity. All treatment options are based on retrospective studies involving a small number of patients. In early-stage disease, untreated follow-up, surgery, involved area radiotherapy, and single-agent rituximab are preferred. Chemoimmunotherapy may be preferred in early disease with high tumor and symptom burden. In advanced-stage disease, radiotherapy can be added to chemotherapy and chemoimmunotherapy. Due to the slow course of the disease, long-term side effects should be observed. Good responses can be achieved in relapsed disease. The risk of transformation is high. It is reasonable to obtain re-biopsies at relapses. Transformed disease and the primary disease have been shown to be clonally related in most cases. Prognosis of transformed disease which is treated with salvage chemo-immunotherapy followed by autologous stem cell transplantation is similar to that of de novo diffuse large B-cell lymphoma. ${ }^{1-3}$ NLPHL patients diagnosed in our clinic are incompatible with the incidence rates stated in the literature. It is suitable to discuss and compare with difference between our data and other data, that has been reported from Turkey.

\section{Conflict of Interests}

Authors declare that there are none.

\section{Acknowledgment}

This study has been presented in $17^{\text {th }}$ Uludag Internal Medicine National Winter Congress, $6^{\text {th }}$ Bursa Family Medicine Association National Congress, $11^{\text {th }}$ Uludag Internal Medicine Nursing Congress, 5-7 March 2021, Bursa, Turkey.

\section{References}

1. Spinner MA, Varma G, Advani RH. Modern principles in the management of nodular lymphocytepredominant Hodgkin lymphoma. Br J Haematol. 2019 Jan;184(1):17-29. doi: 10.1111/bjh.15616.

2. Hartmann S, Eichenauer DA. Nodular lymphocyte predominant Hodgkin lymphoma: pathology, clinical course and relation to T-cell/histiocyte rich large B-cell lymphoma. Pathology. 2020 Jan;52(1):142-53. doi: 10.1016/j.pathol.2019.10.003.

3. Borchmann S, Joffe E, Moskowitz $\mathrm{CH}$, Zelenetz AD, Noy A, Portlock CS, Gerecitano JF, Batlevi CL, Caron PC, Drullinsky P, Hamilton A, Hamlin PA Jr, Horwitz SM, Kumar A, Matasar MJ, Moskowitz AJ, Owens CN, Palomba ML, Younes A, Straus DJ. Active surveillance for nodular lymphocyte-predominant Hodgkin lymphoma. Blood. 2019 May 16;133(20):21219. doi: 10.1182/blood-2018-10-877761. 